Article

\title{
Grain Foods in US Infants Are Associated with Greater Nutrient Intakes, Improved Diet Quality and Increased Consumption of Recommended Food Groups
}

\author{
Yanni Papanikolaou ${ }^{1, *}$ and Victor L. Fulgoni III ${ }^{2}$ (D) \\ 1 Nutritional Strategies, 59 Marriott Place, Paris, ON N3L 0A3, Canada \\ 2 Nutrition Impact, 9725 D Drive North, Battle Creek, MI 49014, USA; vic3rd@aol.com \\ * Correspondence: papanikolaou.yanni@gmail.com; Tel.: +1-519-504-9252
}

Received: 20 September 2019; Accepted: 12 November 2019; Published: 20 November 2019

\begin{abstract}
There are limited data providing guidance on grain foods as part of a healthy dietary pattern in infants and may represent a gap in knowledge for the development of the 2020-2025 Dietary Guidelines for Americans Scientific Advisory Committee report currently in progress. An analysis using infant data from the National Health and Nutrition Examination Survey was conducted to assess grain food relationships with nutrient and energy intakes, diet quality, and food group consumption in infant consumers relative to non-consumers. Grain consumers were defined as infants consuming foods from the main grain food group, as defined by the US Department of Agriculture, and included whole and refined/enriched grains. All infants consuming grain foods had greater energy (kcal) vs. grain non-consumers ( $p^{\prime}$ s $\left.<0.0047\right)$. While infant grain consumers 6- to 12 -months-old $(N=942)$ had higher daily intakes of sodium and added and total sugars, these infants also had significantly higher dietary fiber, calcium, folate, potassium, magnesium, zinc, phosphorus, choline, thiamin, riboflavin, and vitamin $\mathrm{B} 6$ compared to non-consumers. In 13- to 23-month-olds ( $N=1668$ ), grain consumption was associated with greater daily dietary fiber, iron, zinc, magnesium, phosphorus, folate, riboflavin, niacin, thiamin, vitamin A, vitamin B6, and vitamin B12 relative to non-consumers. Diet quality scores were significantly higher in all infant grain consumers examined in comparison to non-consumers $\left(p^{\prime} s<0.0065\right)$. Grain intake was also linked with greater daily intake of several recommended food groups in both younger and older infants versus non-consumption of grains. The current analysis provides evidence to substantiate the inclusion of whole and enriched grain foods as part of the infant dietary pattern as beneficial associations between grain food consumption and dietary quality are apparent. Eliminating and/or reducing grain foods in infant dietary patterns may lead to unintended nutrient and health consequences.
\end{abstract}

Keywords: NHANES; infants; nutrients; grains; diet quality; food groups

\section{Introduction}

Current dietary guidance for Americans acknowledges the robust connection between improved nutrition and diet quality, regular physical activity and maintenance of good health and risk reduction of chronic diseases [1]. Historically, dietary guidance has provided evidence-based nutrition and food-based recommendations for Americans two years of age and older; thus, at present, there are no comprehensive dietary and nutrition recommendations for American children in the first two years of life [2]. In 2012-2013, a partnered initiative between the United States Department of Agriculture (USDA), the US Department of Health and Human Services, and the National Institutes of Health began highlighting gaps in nutrition research, with the objective of generating nutrition guidance and 
recommendations for infants $\leq 2$ years-old, to help guide the development of the upcoming dietary guidance for Americans [3]. Though the 2020-2025 Dietary Guidelines Scientific Advisory Committee is currently convening to generate nutrition and dietary pattern recommendations for Americans from birth into adulthood and the elderly years, there are limited data providing guidance on how grain foods can be incorporated as part of an infant's healthy dietary pattern.

An analysis of usual nutrient intakes using data from the Feeding Infants and Toddlers Study 2016 (FITS 2016) identified approximately 20\% of infants 6- to 11.9-months-old who are at risk for iron inadequacy, while more than $50 \%$ of the same infants had inadequate intake of vitamin $\mathrm{D}$ and vitamin $\mathrm{E}$. Only $3.2 \%$ and $2.5 \%$ of toddlers 12 - to 23.9 -months-old were above the adequate intake recommendation for dietary fiber and potassium, respectively, while many toddlers exceeded the upper limits for sodium, retinol and zinc [4]. While the FITS 2016 study provides data for overall dietary patterns, the contribution of grain food groups continues to be scarce. Data in older children (2-18 years-old) from the National Health and Nutrition Examination Survey (NHANES) 2005-2010 showed that daily dietary fiber intake was significantly higher in several grain food patterns of consumption, including grain foods predominantly from yeast breads, rolls, cereals, and pasta, in comparison to children not including grain foods in their diet. Additionally, iron intakes were significantly higher in all grain food patterns of consumption relative to children not consuming grains [5]. Similarly, national nutrition data on Canadian children from the Canadian Community Health Survey (CCHS-Nutrition 2015) showed that several grain food dietary patterns were associated with beneficial nutrient intakes, while children with limited grain foods in their diet (i.e., $\leq 1$ serving of grains/day) had reduced intakes of fiber, magnesium, zinc, iron, calcium, folate, niacin, riboflavin, and thiamin [6]. Further research in children from the CCHS-Nutrition 2015 identified that intakes of calcium, iron and folate were greater when the diet was comprised of a balanced intake of whole and enriched grain foods, rather than only including one or the other, highlighting the value of a balanced approach to inclusion of grains in the diet and in achieving nutrient recommendations [7]. Recent US data from the NHANES has also shown that certain grains in preschool and school-aged children can contribute meaningful nutrient density in the diet, including greater percentages of dietary fiber, folate, iron, magnesium, zinc, thiamin, riboflavin, vitamin B6, vitamin B12, and vitamin A, relative to energy [8].

With limited evidence addressing nutrient needs and diet quality of infants in the scientific literature, particularly during the growth and development stages, analyses that consider the nutritional contribution of grain foods will help guide the evidence-based nutrition recommendations currently being developed by the Dietary Guidelines Scientific Advisory Committee. Therefore, relative to grain non-consumers, the present analysis aimed to determine grain food consumption in American infants and associations with nutrient and energy intakes, diet quality and food groups.

\section{Experimental Section}

The NHANES is a large database which provides a representative, cross-sectional survey of free-living American residents that is conducted every two years and from which data are released by the National Center for Health Statistics of the Centers for Disease Control and Prevention [9-12]. All required consents were previously obtained for all participants or proxies, and the protocol was approved by the Research Ethics Review Board at the National Center for Health Statistics. For the current analysis, data from eight NHANES datasets (2001-2002; 2003-2004; 2005-2006; 2007-2008; 2009-2010; 2011-2012; 2013-2014 and 2015-2016) were used, which provided data from children less than 2 years of age $[13,14]$, resulting in a robust sample size $(N=3365)$. Nutrient intake data within the current analysis stem from the relevant USDA Food and Nutrient Database for Dietary Studies (FNDDS) [14,15]. Food group data were sourced from the USDA Food Patterns Equivalents Database [16]. The FNDDS contributes energy and nutrient values for all foods and beverages reported in What We Eat in America (WWEIA) [17], the dietary intake component of NHANES.

WWEIA is collected using the USDA's Automated Multiple Pass Method (AMPM), a validated dietary data collection method which has proven to be an evidence-based, efficient and accurate 
format for collecting and assessing dietary intake data for national surveys [18]. AMPM is a fully computerized recall procedure that uses a five-step interview process [18]. Highly-trained interviewers with extensive knowledge of the food and nutrient intake files assess whether collected data is reliable or unreliable $[18,19]$. Previous data lends credibility to the AMPM model and has demonstrated the method's accuracy with intake assessments [20]. While there are two dietary recall days in the NHANES methodology, only Day 1 offers in-person data collection $[17,18]$; hence, the present study only used Day 1 data in the analysis.

\subsection{Subjects}

The current NHANES analysis included all infants 6 to 23 months of age who were classified as either grain consumers or non-consumers (6-12 months: number $[N]$ of consumers $=942 ; N$ of non-consumers $=574 ; 13-23$ months: $N$ of consumers $=1668 ; N$ of non-consumers $=181)$. Study participants were included in the analysis provided they demonstrated reliable and complete 24-hour dietary recalls. Grain consumers were defined as those infants consuming grain foods (with the exclusion of mixed dishes) and breastfeeding during the 24-hour dietary recall as defined by the USDA's Food Patterns Equivalents Database [21].

\subsection{Methods and Statistical Analyses}

Statistical analyses were conducted using SAS software (Version 9.4, SAS Institute, Cary, NC, USA). Appropriate survey parameters were used to generate nationally-representative estimates for infants, which were also appropriately adjusted for the complex design of the NHANES. The USDA food coding system was used to identify and define grain foods. Specifically, as per the Food Patterns Equivalents Database, the grains category consists of two components: whole grains and refined grains. The USDA definition of whole grains encompasses amaranth, barley (not pearled), brown rice, buckwheat, bulgur, millets, oats, popcorn, quinoa, dark rye, triticale, wholegrain cornmeal, whole-grain wheat flour, whole-grain cracked wheat, wild rice, and grain-based products made with $100 \%$ whole grains or their flours. Additionally, refined grains are defined as grains that are degermed or polished and their flours or meal, cornmeal, masa, corn grits, bran of all cereals, cream of rice, cream of wheat, cracked wheat, malted barley or malted flours, pearled barley, rye (light and medium), wheat gluten, and white rice [21].

Least-square means and standard errors of daily energy (calories), nutrient intakes, food group consumption and diet quality were determined for infant grain consumers and non-consumers. Energy intake and diet quality components were adjusted for age, gender, ethnicity, and socioeconomic status (as assessed by the federally established criteria of the poverty income ratio [PIR]) [14]. Nutrient intakes were adjusted for age, gender, ethnicity and PIR, in addition to energy intake. Diet quality was assessed using the USDA's Healthy Eating Index 2015 (HEI-2015), representing the most recent version of the HEI diet quality scale, and considered both the total and sub-component scores. HEI-2015 assesses diet quality and offers a quantitative assessment of conformance to the Dietary Guidelines for Americans, and serves as a tool to measure and track the dietary practices of Americans [22,23]. While the HEI has been created for Americans older than two years of age, most HEI measures are adjusted for energy and, therefore, it was determined that HEI represents an effective and appropriate tool for use in the present research design.

\section{Results}

\subsection{Energy and Nutrients}

Table 1 (6- to 12-month-old infants) and Table 2 (13- to 23-month-old infants) represent adjusted energy and nutrient intakes for grain consumers and non-consumers. While grain consumption was associated with greater intake of energy, sodium and added and total sugars in infants 6- to 12-months of age, intakes of all nutrients were greater in grain consumers than in non-consumers, with the 
exception of intakes of iron, vitamin C, and vitamin E. Contrary to the younger infants, there were no differences in sodium and added and total sugars intake in grain consumers relative to non-consumers. Grain consumption in the older infants was associated with greater daily calories, dietary fiber, iron, zinc, magnesium, phosphorus, riboflavin, folate, thiamin, niacin, vitamin A, vitamin B6, and vitamin $\mathrm{B} 12$ relative to non-consumers of grains.

Table 1. Daily mean energy and nutrient intakes in 6- to 12-month-old grain consumers vs. grain non-consumers.

\begin{tabular}{|c|c|c|c|c|c|c|c|}
\hline \multirow[b]{2}{*}{ Energy/Nutrients } & \multicolumn{2}{|c|}{$\begin{array}{c}\text { GRAIN } \\
\text { NON-CONSUMERS }\end{array}$} & \multicolumn{2}{|c|}{$\begin{array}{c}\text { GRAIN } \\
\text { CONSUMERS }\end{array}$} & \multirow[b]{2}{*}{ Beta } & \multirow[b]{2}{*}{ SE } & \multirow[b]{2}{*}{$p$} \\
\hline & LSMean & SE & LSMean & SE & & & \\
\hline Energy (kcal) & 846 & 16 & 1047 & 22 & 201 & 28 & $<0.0001$ \\
\hline Carbohydrate (g) & 112 & 2 & 138 & 3 & 25 & 4 & $<0.0001$ \\
\hline Added sugars (tsp eq) & 1.0 & 0.15 & 2.8 & 0.17 & 1.8 & 0.22 & $<0.0001$ \\
\hline Total sugars $(\mathrm{g})$ & 80 & 1.3 & 87 & 1.6 & 7 & 2.1 & 0.0029 \\
\hline Protein $(\mathrm{g})$ & 21 & 0.7 & 33 & 1 & 12 & 1.3 & $<0.0001$ \\
\hline Total fat (g) & 35 & 0.8 & 42 & 1.0 & 7 & 1.2 & $<0.0001$ \\
\hline Monounsaturated fatty acids (g) & 11.6 & 0.3 & 14.0 & 0.4 & 2.6 & 0.5 & $<0.0001$ \\
\hline Polyunsaturated fatty acids (g) & 7.2 & 0.2 & 7.7 & 0.2 & 0.5 & 0.3 & 0.0417 \\
\hline Saturated fatty acids (g) & 14.5 & 0.4 & 16.8 & 0.4 & 2.3 & 0.6 & 0.0001 \\
\hline Dietary fiber $(\mathrm{g})$ & 4.5 & 0.2 & 6.6 & 0.2 & 2.2 & 0.3 & $<0.0001$ \\
\hline Calcium (mg) & 720 & 18 & 856 & 22 & 136 & 27 & $<0.0001$ \\
\hline Folate, DFE (mcg) & 193 & 4.6 & 270 & 8.7 & 77 & 10 & $<0.0001$ \\
\hline Iron $(\mathrm{mg})$ & 17.9 & 0.6 & 15.4 & 0.4 & -2.5 & 0.7 & 0.0008 \\
\hline Magnesium (mg) & 109 & 2.8 & 140 & 3.3 & 31 & 4.3 & $<0.0001$ \\
\hline Niacin (mg) & 11.8 & 0.4 & 12.5 & 0.3 & 0.8 & 0.5 & 0.1202 \\
\hline Phosphorus (mg) & 515 & 15 & 730 & 20 & 215 & 25 & $<0.0001$ \\
\hline Potassium (mg) & 1188 & 28 & 1521 & 33 & 333 & 44 & $<0.0001$ \\
\hline Riboflavin (Vitamin B2) (mg) & 1.4 & 0.03 & 1.6 & 0.04 & 0.2 & 0.05 & $<0.0001$ \\
\hline Sodium $(\mathrm{mg})$ & 454 & 27 & 1006 & 44 & 552 & 50 & $<0.0001$ \\
\hline Thiamin (Vitamin B1) (mg) & 1.0 & 0.03 & 1.1 & 0.03 & 0.1 & 0.04 & 0.0047 \\
\hline Total choline $(\mathrm{mg})$ & 131 & 5.4 & 165 & 5.2 & 34 & 7.4 & $<0.0001$ \\
\hline Vitamin A, RAE (mcg) & 717 & 16 & 679 & 12 & 38 & 19 & 0.0458 \\
\hline Vitamin B12 (mcg) & 2.2 & 0.07 & 3.1 & 0.1 & 0.9 & 0.1 & $<0.0001$ \\
\hline Vitamin B6 (mg) & 0.7 & 0.02 & 0.9 & 0.02 & 0.2 & 0.03 & $<0.0001$ \\
\hline Vitamin C (mg) & 106 & 3.2 & 100 & 3.2 & -6.0 & 4.5 & 0.2292 \\
\hline Vitamin D (D2 + D3) (mcg) & 9.06 & 0.2 & 9.08 & 0.2 & 0.02 & 0.2 & 0.9410 \\
\hline Vitamin E as alpha-tocopherol (mg) & 8.4 & 0.2 & 6.9 & 0.2 & -1.5 & 0.3 & $<0.0001$ \\
\hline Zinc $(\mathrm{mg})$ & 6.6 & 0.2 & 7.3 & 0.2 & 0.7 & 0.2 & 0.0037 \\
\hline
\end{tabular}

LSMean = least square mean; SE = standard error; Beta = regression coefficient for difference among groups; Data represent grain consumers (number $[N]=942$ ) and grain non-consumers $(N=574$ ); infants 6- to 12 -months-old, gender combined; National Health and Nutrition Examination Survey [NHANES] 2001-2016; Day 1 intakes.

Table 2. Daily mean energy and nutrient intakes in 13- to 23-month-old grain consumers vs. grain non-consumers.

\begin{tabular}{cccccccc}
\hline & \multicolumn{2}{c}{ GRAIN } & \multicolumn{2}{c}{ GRAIN } & & \\
& NON-CONSUMERS & \multicolumn{2}{c}{ CONSUMERS } & & \\
& LSMean & SE & LSMean & SE & Beta & SE & $p$ \\
\hline Energy/Nutrients & 1166 & 45 & 1309 & 17 & 143 & 50 & 0.0046 \\
Energy (kcal) & 146 & 6 & 173 & 2 & 27 & 7 & 0.0001 \\
Carbohydrate (g) & 7.3 & 0.7 & 7.6 & 0.2 & 0.3 & 0.7 & 0.6423 \\
Added sugars (tsp eq) & 94 & 4.1 & 99 & 1.6 & 5 & 4.1 & 0.2500 \\
Total sugars (g) & 44 & 2 & 49 & 0.7 & 5 & 2.2 & 0.0188 \\
Protein (g) & 46 & 2 & 48 & 1 & 2 & 2.5 & 0.3701 \\
Total fat (g) & 15.9 & 0.8 & 16.0 & 0.3 & 0.1 & 0.9 & 0.8819 \\
Monounsaturated fatty acids (g) & 7.4 & 0.4 & 8.2 & 0.2 & 0.8 & 0.5 & 0.1074 \\
Polyunsaturated fatty acids (g) & 18.8 & 0.9 & 19.7 & 0.4 & 0.9 & 1.0 & 0.3475 \\
Total saturated fatty acids (g) & 6.0 & 0.4 & 8.9 & 0.2 & 2.9 & 0.4 & $<0.0001$ \\
Dietary fiber (g) & 962 & 50 & 1042 & 18 & 80 & 53 & 0.1321 \\
Calcium (mg) & 198 & 12 & 335 & 6.7 & 136 & 14 & $<0.0001$ \\
Folate, DFE (mcg) & & & & & & \\
\hline
\end{tabular}


Table 2. Cont.

\begin{tabular}{cccccccc}
\hline & \multicolumn{2}{c}{ GRAIN } & \multicolumn{2}{c}{ GRAIN } & & & \\
& NON-CONSUMERS & \multicolumn{2}{c}{ CONSUMERS } & & & \\
\hline Energy/Nutrients & LSMean & SE & LSMean & SE & Beta & SE & $p$ \\
\hline Iron (mg) & 8.1 & 0.6 & 9.7 & 0.2 & 1.6 & 0.7 & 0.0197 \\
Magnesium (mg) & 156 & 7.5 & 181 & 2.3 & 25 & 8.0 & 0.0024 \\
Niacin (mg) & 9.4 & 0.5 & 12.2 & 0.2 & 2.8 & 0.5 & $<0.0001$ \\
Phosphorus (mg) & 935 & 44 & 1043 & 15 & 108 & 48 & 0.0256 \\
Potassium (mg) & 1838 & 85 & 1983 & 27 & 145 & 92 & 0.1178 \\
Riboflavin (Vitamin B2) (mg) & 1.7 & 0.08 & 2.0 & 0.03 & 0.3 & 0.09 & 0.0105 \\
Sodium (mg) & 1594 & 145 & 1783 & 27 & 190 & 149 & 0.2063 \\
Thiamin (Vitamin B1) (mg) & 1.0 & 0.05 & 1.1 & 0.02 & 0.1 & 0.05 & 0.0002 \\
Total choline (mg) & 211 & 12.8 & 204 & 3.4 & -7.0 & 14.1 & 0.6318 \\
Vitamin A, RAE (mcg) & 455 & 27 & 564 & 12 & 109 & 30 & 0.0004 \\
Vitamin B12 (mcg) & 3.7 & 0.2 & 4.3 & 0.1 & 0.6 & 0.2 & 0.0107 \\
Vitamin B6 (mg) & 0.9 & 0.05 & 1.2 & 0.02 & 0.3 & 0.05 & $<0.0001$ \\
Vitamin C (mg) & 81 & 9.2 & 83 & 2.5 & 2.0 & 9.0 & 0.8149 \\
Vitamin D (D2 + D3) (mcg) & 8.6 & 0.5 & 8.4 & 0.2 & 0.2 & 0.6 & 0.7625 \\
Vitamin E as alpha-tocopherol (mg) & 4.0 & 0.3 & 3.9 & 0.1 & 0.1 & 0.4 & 0.7889 \\
Zinc (mg) & 6.3 & 0.3 & 7.5 & 0.1 & 1.2 & 0.3 & 0.0005 \\
\hline
\end{tabular}

LSMean = least square mean; SE = standard error; Beta = regression coefficient for difference among groups; Data represent grain consumers $(N=1,668)$ and grain non-consumers $(N=181)$; infants 13- to 23-months-old, gender combined; NHANES 2001-2016; Day 1 intakes.

\subsection{Diet Quality: USDA Healthy Eating Index-2015 Total and Sub-Component Scores}

Mean total and sub-component scores in infants for the HEI are displayed in Table 3 (6- to 12-month-old infants) and Table 4 (13- to 23-month-old infants). Younger grain consuming infants had significantly lower scores for sodium, but also had greater scores for greens and beans, total fruit, whole grains, refined grains, dairy foods, total protein foods, seafood and plant protein foods, and saturated fat in comparison to non-consumers of grains. Older infants consuming grains had greater scores for total fruit, whole fruit, whole grains, and refined grains relative to non-consumers. No differences were observed for protein foods or sodium scores between consumers and non-consumers. Grain consumers in both age groups had significantly higher total HEI scores relative to infant non-consumers.

Table 3. Mean (standard error [SE]) total Healthy Eating Index-2015 and sub-component scores for 6to 12-month-old grain consumers vs. grain non-consumers.

\begin{tabular}{|c|c|c|c|c|c|}
\hline \multirow[b]{2}{*}{ HEI Scores } & \multicolumn{2}{|c|}{ GRAIN NON-CONSUMERS } & \multicolumn{2}{|c|}{ GRAIN CONSUMERS } & \multirow[b]{2}{*}{$p$} \\
\hline & LSMean & SE & LSMean & SE & \\
\hline Component 1: Total vegetables & 2.08 & 0.1 & 2.09 & 0.1 & 0.9768 \\
\hline Component 2: Greens and beans & 0.24 & 0.1 & 0.61 & 0.1 & $<0.0001$ \\
\hline Component 3: Total fruit & 3.24 & 0.1 & 3.60 & 0.1 & 0.0079 \\
\hline Component 4: Whole fruit & 3.11 & 0.1 & 3.31 & 0.1 & 0.1916 \\
\hline Component 5: Whole grains & 1.91 & 0.2 & 2.63 & 0.1 & 0.0012 \\
\hline Component 6: Dairy & 1.71 & 0.2 & 4.45 & 0.2 & $<0.0001$ \\
\hline Component 7: Total protein foods & 0.90 & 0.1 & 1.88 & 0.1 & $<0.0001$ \\
\hline Component 8: Seafood and plant protein & 0.23 & 0.1 & 0.65 & 0.1 & $<0.0001$ \\
\hline Component 9: Fatty acid ratio & 2.02 & 0.1 & 1.96 & 0.1 & 0.6418 \\
\hline Component 10: Sodium & 9.66 & 0.1 & 8.72 & 0.1 & $<0.0001$ \\
\hline Component 11: Refined grains & 9.62 & 0.1 & 8.90 & 0.1 & $<0.0001$ \\
\hline Component 12: Saturated fat & 2.30 & 0.2 & 2.92 & 0.2 & 0.0033 \\
\hline HEI-2015 TOTAL SCORE & 46.73 & 0.5 & 51.2 & 0.4 & $<0.0001$ \\
\hline
\end{tabular}

LSMean = least square mean; SE = standard error; Beta = regression coefficient for difference among groups; Data represent grain consumers $(N=942)$ and grain non-consumers $(N=574)$; infants aged 6- to 12-months-old, gender combined; NHANES 2001-2016; Day 1 intakes. 
Table 4. Mean (SE) total and sub-component scores for the Healthy Eating Index 2015 (HEI-2015) in 13to 23-month-old grain consumers vs. grain non-consumers.

\begin{tabular}{cccccc}
\hline & \multicolumn{2}{c}{ GRAIN NON-CONSUMERS } & \multicolumn{3}{c}{ GRAIN CONSUMERS } \\
\hline HEI Scores & LSMean & SE & LSMean & SE & $p$ \\
\hline Component 1: Total vegetables & 1.97 & 0.2 & 2.01 & 0.1 & 0.8504 \\
Component 2: Greens and beans & 0.99 & 0.2 & 0.87 & 0.1 & 0.5718 \\
Component 3: Total fruit & 3.40 & 0.2 & 3.94 & 0.1 & 0.0143 \\
Component 4: Whole fruit & 2.68 & 0.2 & 3.30 & 0.1 & 0.0143 \\
Component 5: Whole grains & 1.00 & 0.2 & 2.54 & 0.1 & 0.0090 \\
Component 6: Dairy & 8.47 & 0.4 & 8.98 & 0.1 & 0.2296 \\
Component 7: Total protein foods & 3.19 & 0.2 & 3.00 & 0.1 & 0.2955 \\
Component 8: Seafood and plant protein & 1.36 & 0.2 & 1.26 & 0.1 & 0.6996 \\
Component 9: Fatty acid ratio & 1.89 & 0.3 & 1.69 & 0.1 & 0.5499 \\
Component 10: Sodium & 6.76 & 0.3 & 6.78 & 0.1 & 0.9672 \\
Component 11: Refined grains & 8.56 & 0.3 & 7.56 & 0.1 & 0.0030 \\
Component 12: Saturated fat & 2.97 & 0.4 & 3.71 & 0.1 & 0.0539 \\
HEI-2015 TOTAL SCORE & 51.00 & 1.0 & 53.76 & 0.4 & 0.0064 \\
\hline
\end{tabular}

LSMean = least square mean; SE $=$ standard error; Data represent grain consumers $(N=1,668)$ and grain non-consumers ( $N=181$ ); infants aged 13- to 23-months-old, gender combined; NHANES 2001-2016; Day 1 intakes; Covariates include age, gender, ethnicity, and poverty income ratio.

\subsection{Food Group Intake in Infants}

Mean food group intake in infants for grain consumers and grain non-consumers is shown in Table 5 (6-12-month-old infants) and Table 6 (13-23-month-old infants). Infants 6-12 months-old had significantly higher intakes of milk, cheese, and total dairy foods compared to grain non-consumers. Infant grain consumption was linked to higher refined and whole grain intake, as well as greater intake in terms of total fruits, total vegetables and total meat, poultry, seafood, nuts and seeds compared to non-consumers of grains.

No differences between grain consumers and non-consumers were seen for milk, yogurt, total dairy foods, total vegetables, and total meat, poultry, seafood, nuts and seeds in infants 13-23 months of age. However, grain consumers in this age group had higher daily intakes of cheese, refined and whole grains, and total fruits versus grain non-consumers.

Table 5. Mean (SE) food group intake for 6- to 12-month-old grain consumers vs. grain non-consumers.

\begin{tabular}{cccccc}
\hline & \multicolumn{2}{c}{ GRAIN NON-CONSUMERS } & \multicolumn{3}{c}{ GRAIN CONSUMERS } \\
\hline Food Group & LSMean & SE & LSMean & SE & $p$ \\
\hline Cheese (cup eq) & 0.04 & 0.01 & 0.16 & 0.02 & $<0.0001$ \\
Milk (cup eq) & 0.29 & 0.05 & 1.06 & 0.08 & $<0.0001$ \\
Yogurt (cup eq) & 0.03 & 0.01 & 0.05 & 0.01 & 0.2249 \\
Total Dairy (cup eq) & 0.37 & 0.06 & 1.27 & 0.09 & $<0.0001$ \\
Refined Grains (oz eq) & 0.71 & 0.05 & 1.67 & 0.10 & $<0.0001$ \\
Whole Grains (oz eq) & 0.26 & 0.02 & 0.42 & 0.02 & $<0.0001$ \\
Total Fruits (cup eq) & 0.67 & 0.03 & 0.99 & 0.04 & $<0.0001$ \\
Total Vegetables * (cup eq) & 0.42 & 0.02 & 0.48 & 0.02 & 0.0475 \\
Total Meat, Poultry, Seafood, & 0.43 & 0.05 & 1.10 & 0.07 & $<0.0001$ \\
Nuts \& Seeds (oz eq) & & & & &
\end{tabular}

LSMean = least square mean; SE = standard error; Data represent grain consumers $(N=942)$ and grain non-consumers $(N=574)$; infants aged 6- to 12-months-old, gender combined; NHANES 2001-2016; Day 1 intakes. * excludes legumes. 
Table 6. Mean (SE) food group intake for 13- to 23-month-old grain consumers vs. grain non-consumers.

\begin{tabular}{cccccc}
\hline & \multicolumn{2}{c}{ GRAIN NON-CONSUMERS } & \multicolumn{3}{c}{ GRAIN CONSUMERS } \\
\hline Food Group & LSMean & SE & LSMean & SE & $p$ \\
\hline Cheese (cup eq) & 0.26 & 0.04 & 0.39 & 0.02 & 0.0011 \\
Milk (cup eq) & 2.10 & 0.17 & 2.25 & 0.05 & 0.3979 \\
Yogurt (cup eq) & 0.07 & 0.02 & 0.09 & 0.01 & 0.3480 \\
Total Dairy (cup eq) & 2.46 & 0.18 & 2.74 & 0.06 & 0.1258 \\
Refined Grains (oz eq) & 2.06 & 0.29 & 2.90 & 0.06 & 0.0053 \\
Whole Grains (oz eq) & 0.19 & 0.04 & 0.51 & 0.02 & $<0.0001$ \\
Total Fruits (cup eq) & 1.15 & 0.11 & 1.41 & 0.04 & 0.0349 \\
Total Vegetables * (cup eq) & 0.49 & 0.05 & 0.55 & 0.02 & 0.2316 \\
Total Meat, Poultry, Seafood, & 2.13 & 0.18 & 2.09 & 0.05 & 0.8134 \\
Nuts \& Seeds (oz eq) & & & & &
\end{tabular}

LSMean = least square mean; SE = standard error; Data represent grain consumers $(N=1668)$ and grain non-consumers ( $N=181)$; infants aged 13- to 23-months-old, gender combined; NHANES 2001-2016; Day 1 intakes. * excludes legumes.

\section{Discussion}

At present, there are limited data considering dietary patterns of infants, with even less evidence available pertaining to the role of grain foods within this population. Based on our review of the literature, this is the first study in infants using data from NHANES to examine grain consumption and associations with nutrient intake, diet quality and food group intake. Grain food consumption in 6- to 23-month-olds was found to be associated with higher nutrient intakes, better diet quality scores, and increased amounts of foods to encourage when compared to grain non-consumers. Specifically, grain consumption in 6- to 12-month-olds was linked with greater daily intake of numerous essential nutrients relative to non-consumers. Similarly, grain consumption in older infants was associated with greater daily intake of dietary fiber, folate, iron, magnesium, niacin, phosphorus, riboflavin, thiamin, vitamin A, vitamin B12, vitamin B6 and zinc compared to non-consumers. While grain consumption was associated with significantly greater intake of sodium and added and total sugars in infants 6- to 12-months-old, the 13- to 23-month-old infants showed no differences in sodium or added and total sugars intake when comparing grain consumers to non-consumers. Grain consumers of both infant age groups had significantly better diet quality, as reflected by the higher total HEI scores, relative to non-consumers. This may be attributed to the scores contributed from greater intake of greens and beans, total fruit, whole grains, dairy foods, total protein, seafood and plant protein foods in younger infants, and greater intake of total fruit, whole fruit and whole grains in the older infants, when compared to the grain non-consumers. When assessing food group intake, younger infants tended to eat greater amounts of dairy foods, whole grains, refined grains, total fruits, total vegetables and protein-rich foods, while older infants had greater daily intake of cheese, refined grains, whole grains and total fruit, relative to non-consumers.

While our previous work did not consider the contribution of grain foods in infants, grain consumption in young children appeared to be beneficial, with grain foods acting as part of a healthy dietary pattern. Indeed, several types of grain foods, including refined and whole grains, contributed to nutrient density in US children aged 1- to 3-years. Specifically, grain foods accounted for approximately $13 \%$ of all calories, $16 \%$ of sodium, $7 \%$ of total sugar and $5 \%$ of saturated fat in the total diet, while providing about $23 \%$ of all dietary fiber, $40 \%$ of folate, $38 \%$ of iron, $30 \%$ of thiamin, $29 \%$ of niacin, $23 \%$ of vitamin $\mathrm{B} 6,19 \%$ of zinc, $18 \%$ of vitamin $\mathrm{A}, 18 \%$ of riboflavin, $17 \%$ of vitamin $\mathrm{B} 12$, and $13 \%$ of magnesium. Subcategories of grain foods, including breads, rolls, tortillas, and ready-to-eat cereals also contributed meaningful levels of nutrients, demonstrating that certain grain foods contribute nutrient density that surpasses caloric contributions in the diet [5]. Similarly, recent work using data from the NHANES 2015-2016 found that ready-to-eat cereal consumption in children aged 6 months to 17 years was associated with greater daily intake of carbohydrates, total sugar, dietary fiber, calcium, 
zinc, magnesium, iron, potassium, folate, niacin, riboflavin, thiamin, vitamin A, vitamin B6, vitamin B12, and vitamin D compared to non-consumers of ready-to-eat cereals. Dietary folate, thiamin, and vitamin B6 daily intakes were significantly greater in infant ready-to-eat cereal consumers relative to non-consumers [24]. Similar to the current findings, children aged 6-months to 17-years-old consuming ready-to-eat cereals showed significantly elevated intake of food groups to encourage, including $29 \%$ greater total dairy intake and $61 \%$ higher whole grain intake compared to non-consumers. Intake of milk and whole grains were significantly higher in infants and toddlers consuming ready-to-eat cereals when compared to the infant and toddler non-consumers [24], suggesting that grain foods may be a delivery vehicle for nutrient-rich dairy foods.

While the NHANES analysis in infant and toddler ready-to-eat cereal consumers did not find significant differences in energy intake [24], the current analysis showed that energy intake in grain consumers was consistently higher relative to non-consumers. Likewise, while the infant and toddler study showed no differences in sodium intake between ready-to-eat cereal consumers and non-consumers, the current analysis showed higher sodium intakes in grain consumers 6- to 12-months-old relative to non-consumers. This has also been documented in previously published work in preschool and school-aged children in the US and Canada [5,6]. Therefore, while grain foods can be contributors of energy and sodium to the diet of children, early acceptance and familiarity with nutrient-dense grain foods will likely help close nutrient intake recommendation gaps as children grow and develop into adulthood, with particular emphasis on Dietary Guidelines for Americans shortfall nutrients and nutrients of public health concern, which include, but are not limited to, dietary fiber, folate, magnesium, calcium, and iron [1].

The current study has limitations inherent in cross-sectional and/or observational research which have previously been reported. Importantly, the present results are observations between grain consumers, nutrient intakes, diet quality and food group consumption. Thus, the current observational research cannot be used to establish cause and effect. As the current findings are reliant on self-reported methods, this can potentially lead to under- or over-estimation of food intake, which can contribute inaccuracies in energy and nutrient intakes. Dietary recalls in infant research also rely on the memory of the parents or caregivers, and while validated procedures are established to gather nutrition data, bias from memory recall challenges may be introduced into the analysis [25]. Also, the current study evaluated dietary patterns with and without grain foods. Thus, other foods comprising the overall infant dietary pattern are likely to contribute to the observed associations. A substantial advantage of using the NHANES data includes a robust nationally-representative sample of American children with corresponding data on energy and nutrient intakes, as well as food and beverage consumption.

\section{Conclusions}

This is the first study to demonstrate differences in intakes of nutrients, diet quality and food group consumption between grain consumers and non-consumers in US infants. Grain consumption, in general, was associated with higher nutrient intakes, better diet quality scores, and increased amounts of foods to encourage when compared to grain non-consumers. Infants 6- to 12-month-olds had higher daily intake of sodium and added and total sugar, but also had significantly higher daily intake of dietary fiber, calcium, folate, magnesium, phosphorus, potassium, riboflavin, thiamin, choline, zinc and vitamin B6 relative to non-consumers. In addition, 13- to 23-month-old participants had higher daily intake of dietary fiber, folate, iron, magnesium, niacin, phosphorus, riboflavin, thiamin, vitamin A, vitamin B12, vitamin B6 and zinc. An investigation of food group intake showed that younger grain-consuming infants tended to eat greater amounts of several recommended food groups, including higher amounts of dairy foods, whole grains, total fruits, total vegetables and protein-rich foods, while older grain-consuming infants had greater daily intake of cheese, whole grains and total fruit compared to grain non-consumers. The current evidence supports the inclusion of grain foods in infant dietary patterns. Furthermore, eliminating or reducing grain foods in the diets of American infants 6-23 months-old may have unintended nutrient, food group and diet quality consequences in 
the future. While staying within caloric needs and recommendations, and being sensitive to added sugars, saturated fat, and sodium intake, caregivers are encouraged to select whole and enriched grain foods that contribute nutrient density.

Author Contributions: Y.P. and V.L.F. provided on the intellectual knowledge and interpretation of the research findings; V.L.F. designed the research methodology and produced the final analyses; Y.P. developed and finalized the manuscript, and both Y.P. and V.L.F. provided approval of the final manuscript incorporating the study analysis.

Funding: This research was funded by the Grain Foods Foundation.

Acknowledgments: Grain Foods Foundation in Washington, DC, provided funding support for the current research.

Conflicts of Interest: Grain Foods Foundation had no role in the study design, nor in the collection, analysis, or interpretation of the data. Grain Foods Foundation also had no input in the writing of the manuscript and in the decision to publish the results. Y.P. provides nutrition and regulatory science consulting for private and public groups and collaborates with V.L.F. on database analyses. V.L.F. provides nutrition science consulting for various private and public groups.

\section{References}

1. United States Department of Health and Human Services and United States Department of Agriculture. 2015-2020 Dietary Guidelines for Americans, 8th ed.; U.S. Government Printing Office: Washington, DC, USA, 2015. Available online: https://health.gov/dietaryguidelines/2015/resources/2015-2020_Dietary_Guidelines. pdf (accessed on 15 August 2019).

2. United States Department of Agriculture. Food and Nutrition Service. Pregnancy and Birth to 24 Months. Research, Analysis and Background. Available online: https://www.fns.gov/resources/pregnancy-and-birth24-months (accessed on 9 September 2019).

3. United States Department of Agriculture. Center for Nutrition Policy and Promotion. Pregnancy and Birth to 24 Months Project. Available online: https://www.cnpp.usda.gov/birthto24months (accessed on 31 August 2019).

4. Bailey, R.L.; Catellier, D.J.; Jun, S.; Dwyer, J.T.; Jacquier, E.F.; Anater, A.S.; Eldridge, A.L. Total usual nutrient intakes of US children (under 48 months): Findings from the Feeding Infants and Toddlers Study (FITS) 2016. J. Nutr. 2018, 148, 1557-1566. [CrossRef] [PubMed]

5. Papanikolaou, Y.; Jones, J.M.; Fulgoni, V.L. Several grain dietary patterns are associated with better diet quality and improved shortfall nutrient intakes in US children and adolescents: A study focusing on the 2015-2020 Dietary Guidelines for Americans. Nutr. J. 2017, 16, 13. [CrossRef] [PubMed]

6. Hosseini, S.H.; Papanikolaou, Y.; Isalm, N.; Rashmi, P.; Shamloo, A.; Vatanparast, H. Consumption patterns of grain-based foods among children and adolescents in Canada: Evidence from Canadian Community Health Survey-Nutrition 2015. Nutrients 2019, 11, 623. [CrossRef] [PubMed]

7. Hosseini, S.H.; Jones, J.M.; Vatanparast, H. Association between grain intake, nutrient intake, and diet quality of Canadians: Evidence from the Canadian Community Health Survey-Nutrition 2015. Nutrients 2019, 11, 1937. [CrossRef] [PubMed]

8. Papanikolaou, Y.; Fulgoni, V.L. Certain grain foods can be meaningful contributors to nutrient density in the diets of U.S. children and adolescents: Data from the National Health and Nutrition Examination Survey, 2009-2012. Nutrients 2017, 9, 160. [CrossRef] [PubMed]

9. Centers for Disease Control and Prevention. National Center for Health Statistics. National Health and Nutrition Examination Survey. Available online: https://www.cdc.gov/nchs/nhanes/index.htm. (accessed on 21 August 2019).

10. Ahuja, J.K.A.; Montville, J.B.; Omolewa-Tomobi, G.; Heendeniya, K.Y.; Martin, C.L.; Steinfeldt, L.C.; Anand, J.; Adler, M.E.; LaComb, R.P.; Moshfegh, A.J. USDA Food and Nutrient Database for Dietary Studies, 5.0-Documentation and User Guide; U.S. Department of Agriculture, Agricultural Research Service, Food Surveys Research Group: Beltsville, MD, USA, 2012.

11. US Department of Agriculture. Agricultural Research Service. Food Data Central. Available online: https://fdc.nal.usda.gov/ (accessed on 21 October 2019).

12. Ahuja, J.K.; Moshfegh, A.J.; Holden, J.M.; Harris, E. USDA food and nutrient databases provide the infrastructure for food and nutrition research, policy, and practice. J. Nutr. 2013, 143, 241S-249S. [CrossRef] [PubMed] 
13. Centers for Disease Control and Prevention. National Center for Health Statistics. National Health and Nutrition Examination Survey. Analytic and Reporting Guidelines. Available online: https://wwwn.cdc.gov/ nchs/nhanes/analyticguidelines.aspx (accessed on 20 August 2019).

14. National Center for Health Statistics. Centers for Disease Control and Prevention. US Department of Health and Human Services. US National Health and Nutrition Examination Survey, 2015-2016. Available online: https://wwwn.cdc.gov/nchs/nhanes/ContinuousNhanes/Default.aspx?BeginYear=2015 (accessed on 16 September 2019).

15. Rhodes, D.G.; Adler, M.E.; Clemens, M.E.; Moshfegh, A.J. What we eat in America food categories and changes between survey cycles. J. Food Comp. Anal. 2017, 64, 107-111. [CrossRef]

16. Overview of Food Patterns Equivalent Database. Available online: https://www.ars.usda.gov/northeast-area/ beltsville-md-bhnrc/beltsville-human-nutrition-research-center/food-surveys-research-group/docs/fpedoverview/ (accessed on 23 August 2019).

17. United States Department of Agriculture. Agricultural Research Service. Food Service Research Group: Beltsville, MD. What We Eat in America, National Health and Nutrition Examination Survey Overview, 2011-2014: Data Collection. Available online: https://www.ars.usda.gov/northeast-area/beltsville-md-bhnrc/ beltsville-human-nutrition-research-center/food-surveys-research-group/docs/wweianhanes-overview/ (accessed on 26 August 2019).

18. Centers for Disease Control and Prevention. National Center for Health Statistics. National Health and Nutrition Examination Survey 2013-2014. Data Documentation, Codebook, and Frequencies. Dietary Interview-Total Nutrient Intakes, First Day (DR1TOT_H). 2016. Available online: https://wwwn.cdc.gov/ Nchs/Nhanes/2013-2014/DR1TOT_H.htm (accessed on 2 September 2019).

19. Raper, N.; Perloff, B.; Ingwersen, L.; Steinfeldt, L.; Anand, J. An overview of USDA's dietary intake data system. J. Food Compos. Anal. 2004, 17, 545-555. [CrossRef]

20. Moshfegh, A.J.; Rhodes, D.G.; Baer, D.J.; Murayi, T.; Clemens, J.C.; Rumpler, W.V.; Paul, D.R.; Sebastian, R.S.; Kuczynski, K.C.; Ingwersen, L.A.; et al. The USDA Automated Multiple-Pass Method reduces bias in the collection of energy intakes. Am. J. Clin. Nutr. 2008, 88, 324-332. [CrossRef] [PubMed]

21. Bowman, S.A.; Clemens, J.C.; Friday, J.E.; Lynch, K.L.; Moshfegh, A.J. Food Patterns Equivalents Database 2013-2014: Methodology and User Guide [Online]. In Food Surveys Research Group, Beltsville Human Nutrition Research Center, Agricultural Research Service; U.S. Department of Agriculture: Beltsville, MD, USA, 2017. Available online: http://www.ars.usda.gov/nea/bhnrc/fsrg (accessed on 2 September 2019).

22. Krebs-Smith, S.M.; Pannucci, T.E.; Subar, A.F.; Kirkpatrick, S.I.; Lerman, J.L.; Tooze, J.A.; Wilson, M.M.; Reedy, J. Update of the Healthy Eating Index: HEI-2015. J. Acad. Nutr. Diet. 2018, 118, 1591-1602. [CrossRef] [PubMed]

23. Panizza, C.E.; Shvetsov, Y.B.; Harmon, B.E.; Wilkens, L.R.; Le Marchand, L.; Haiman, C.; Reedy, J.; Boushey, C.J. Testing the predictive validity of the Healthy Eating Index-2015 in the multiethnic cohort: Is the score associated with a reduced risk of all-cause and cause-specific mortality? Nutrients 2018, 10, 452. [CrossRef] [PubMed]

24. Smith, J.D.; Zhu, Y.; Vanage, V.; Jain, N.; Holschuh, N.; Hermetet Agler, A. Association between ready-to-eat cereal consumption and nutrient intake, nutritional adequacy, and diet quality among infants, toddlers, and children in the National Health and Nutrition Examination Survey 2015-2016. Nutrients 2019, 11, 1989. [CrossRef] [PubMed]

25. Dwyer, J.; Picciano, M.F.; Raiten, D.J. Members of the Steering Committee. Collection of food and dietary supplement intake data: What we eat in America-NHANES. J. Nutr. 2003, 133, 590-600. [CrossRef] [PubMed]

(C) 2019 by the authors. Licensee MDPI, Basel, Switzerland. This article is an open access article distributed under the terms and conditions of the Creative Commons Attribution (CC BY) license (http://creativecommons.org/licenses/by/4.0/). 